\begin{abstract}
Phenolic resins are used in many aspects of everyday life, e.g. as the matrix material for carbon fibre laminates used in the aerospace industry. Consequently detailed knowledge of this material, especially while under shock loading, is extremely useful for the design of components that could be subjected to impact during their lifespan. The shock Hugoniot equation of state for phenolic resin (Durite SC-1008), with initial density of 1.18 $\mathrm{gcm}^{-3}$ have been determined using the plate-impact technique with in situ manganin stress gauges. The Hugoniot equation in the shock velocity-particle velocity plane was found to be non-linear in nature with the following equation: $\mathrm{U}_{S}=2.14+3.79 \mathrm{u}_{p}-1.68 \mathrm{u}_{p}^{2}$. Further, the Hugoniot in the pressure-volume plane was observed to largely follow the hydrostatic curve. Lateral gauge measurements were also obtained. An ANSYS Autodyn ${ }^{\text {TM }}$ 2D model was used to investigate the lateral stress behaviour of the SC-1008. A comparison of the Hugoniot elastic limit calculated from the shear strength and measured sound speeds gave reasonable agreement with a value of $0.66 \pm 0.35 \mathrm{GPa}$ obtained.
\end{abstract}




\title{
Shock Behaviour of a Phenolic Resin
}

\author{
David C. Wood • Paul J. Hazell • Gareth J. Appleby-Thomas • Nick R. \\ Barnes
}

\section{Introduction}

Phenolic resins have a wide range of applications which include, but are not exclusive to, electrical insulation, kitchen utensils [1] and use in heat-shields for the aerospace industry [2]. Phenolic resins are used in such applications due to their resistance to abrasion and dimensional stability over a range of operating temperatures, with this latter property due to the fact that phenolic resins are thermosetting plastics. The use of phenolic resins in the aerospace industry and the consequent possibility of impact damage, reinforces the requirement for knowledge of shock propagation in such materials.

Many methods can be used for generating shock in materials including contact explosives [3], laser ablation [4] as well as plate impact technique [5] employed here. Five key parameters are needed to understand the behaviour of shocked materials. These are shock velocity $\mathrm{U}_{S}$, particle velocity $\mathrm{u}_{p}$, density $\rho$, pressure $\mathrm{P}$ and internal energy E. Usefully, only two parameters are needed to fully define a shock. The Rankine-Hugoniot equations [6], based on the principles of conservation of energy, mass and momentum then allow the other three parameters to be calculated. The parameters measured in this study were $\mathrm{U}_{S}$ and $\sigma_{x}$, with $\mathrm{u}_{p}$ found using the impedance matching technique [6], while density was calculated from the Rankine-Hugoniot equations. This allowed the Hugoniots in the $\mathrm{U}_{S}-\mathrm{u}_{p}$ and $\mathrm{P}-\mathrm{V}$ planes to be ascertained.

Hugoniot relationships in the $\mathrm{U}_{S}-\mathrm{u}_{p}$ plane are generally linear in nature following Eq. (1), where $c_{0}$ is the

D. C. Wood, P. J. Hazell and G. J. Appleby-Thomas Cranfield University, Cranfield Defence and Security, Shrivenham, Swindon, SN6 8LA

E-mail: d.wood@cranfield.ac.uk

N. R. Barnes

AWE, Aldermaston, Reading, Berkshire, RG7 4PR intercept at zero particle velocity and $\mathrm{S}$ is the resultant slope of the Hugoniot. However, non-linear relationships do occur $[7,8]$. In such cases a second order quadratic equation, of the form shown in Eq. (2), may be employed. Such non linear equations of state have also been used in computational simulations of polymers, e.g. Ref. [9].

$U_{S}=c_{0}+S u_{p}$
$U_{S}=c_{0}+S_{1} u_{p}+S_{2} u_{p}^{2}$

The hydrostatic pressure $\mathrm{P}_{H}$ due to a shock in a material is given by Eq. (3) which can be written in the form of Eq. (4) for the pressure-volume plane, where $\rho_{0}$ is the initial density with $v$ and $v_{o}$ being the volume at a given pressure and initial volume respectively. The Hugoniot in the $\mathrm{U}_{S}-\mathrm{u}_{p}$ plane is used to calculate the value of $\mathrm{P}_{H}$.

$P_{H}=\rho_{0} U_{S} u_{p}$

$P_{H}=\rho_{0} U_{S}^{2}\left(1-\frac{v}{v_{0}}\right)$

In addition, the hydrostatic $\left(\mathrm{P}_{H}\right)$ and deviatoric $(\tau)$ elements of stress are linked by Eq. (5). This equation implies that if the longitudinal stress equals the hydrostatic pressure then there is little to no strength effect in the material.

$\sigma_{x}=P_{H}+\frac{4}{3} \tau$

With polymers the depth of knowledge with regards to the shock response varies greatly, from materials that are well defined e.g. polymethylmethacrylate (PMMA) $[7,8]$, to materials that have been little studied. Further, it has been shown that different samples of the same polymer can have different shock Hugoniot profiles due to slightly different compositions or manufacturing processes. Such behaviour is clearly exhibited by 
the abundant scatter of data noticed by Barker and Hollenbach in PMMA [7].

Carter and Marsh [8] studied the shock response of 20 polymers. For all polymeric materials studied they consistently found a discrepancy between the zero pressure intercept of the $\mathrm{U}_{S}-\mathrm{u}_{p}$ curve and the ultrasonically measured sound speeds. They attributed this phenomena to the forces between adjacent polymer chains being orders of magnitude lower than the forces along the backbone of the polymer, making initial compression two-dimensional in nature. Such behaviour was shown to lead to non-linearity in the $\mathrm{U}_{S}-\mathrm{u}_{p}$ plane. The subsequent linear behaviour of the Hugoniot at elevated particle velocities was thought to represent the threedimensional compressive behaviour. It was also found that a phase change occurred in many of the polymers investigated in the 20-30 GPa range.

In the literature there is limited knowledge on the shock behaviour of phenolic resins with most studies concerned with phenolic resins as part of a composite system. Carter and Marsh, however, did include the phenolic resin Durite HR 300-Borden in their investigations.

The dynamic response of epoxy-resins has been more extensively studied [8,10-12]. Munson and May [10] investigated the effect of different hardeners which resulted in different structure along with different levels of cross linking. They found that in the $\mathrm{U}_{S}-\mathrm{u}_{p}$ plane a single equation could explain the behaviour. In the pressure-volume plane there was no noticeable discrepancy between the epoxies with different hardeners over the investigated range which reached values of $2 \mathrm{GPa}$.

Another epoxy resin RTM-6, used as the matrix component in aerospace-grade carbon fibre composites, was studied by Hazell et al. [11]. Its dynamic behaviour was found to be comparable to that of other epoxy resins. Furthermore deviation from the hydrostatic pressure was seen in RTM-6 above 4 GPa. In other work, Appleby-Thomas et al. [12] investigated the lateral stress behaviour of RTM-6. The Hugoniot elastic limit and dynamic yield strength for RTM-6 was found to be comparable to other epoxy resins.

Millett et al. [13] investigated the shock behaviour of a composite which included different ratios of alumina particulates in an epoxy resin. As alumina particulates were used the sample was considered to be isotropic in nature allowing for a simplified analysis. It was found that by increasing the ratio of alumina particles in the epoxy, the sample material started to behave similarly to a metal or ceramic.

In this work the dynamic shock behaviour of a phenolic resin SC-1008 was investigated using manganin stress gauges. These gauges measure the longitudinal

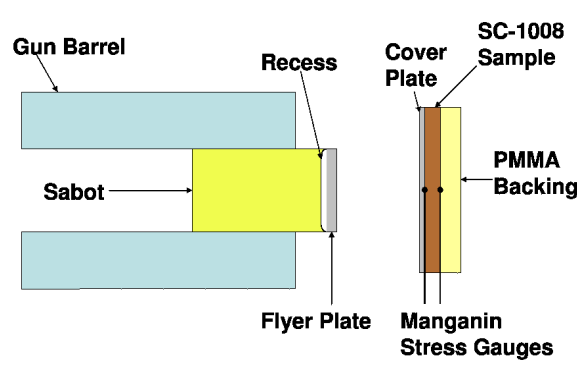

Fig. 1 General experiment setup

and lateral stresses induced in the samples. From this data $\mathrm{U}_{S}-\mathrm{u}_{p}$ and $\mathrm{P}-\mathrm{V}$ Hugoniot relationships were derived.

\section{Experimental Techniques}

The experimental method employed for this investigation was the plate impact technique. This involved using a $\varnothing 50 \mathrm{~mm}, 5 \mathrm{~m}$ barrel, single-stage gas gun [5] which accelerates a flyer plate into a target material, with all the surfaces perpendicular to the direction of impact machined flat and parallel to $\pm 5 \mu \mathrm{m}$. The experimental setup for the gun, also showing a typical target configuration, is shown in Fig. 1. The flyer and cover plate were made of the same material, usually Aluminium (Dural) or Copper, employed due to their well characterised nature e.g. [14]. Further, the target is attached to a sacrificial barrel extension. Taken together these measures ensured that any misalignment on impact was consistently less than $1.5 \mathrm{mrad}$ [15].

Vishay Micro-measurements manganin stress gauges of types LM-SS-125CH-048 and J2M-SS-580SF-025 were employed for longitudinal and lateral shots respectively. Longitudinal gauges measure the longitudinal stress exerted on the material whereas the lateral gauges give a stress which is dependent on the strength of the sample [16]. These gauges were used due to their stable behaviour over a wide range of temperatures. As stress is exerted on the manganin gauge element a change in resistance of the gauge occurs. Using a calibration technique detailed by Rosenberg et al. [17] for longitudinal gauges and Rosenberg et al. $[18,19]$ and Millett et al. [20] for lateral gauges, this allows calculation of inmaterial stress. The longitudinal gauges were protected by layers of $25 \mu \mathrm{m}$ thick Mylar ${ }^{\circledR}$, which also insulated them from the cover plate. However, the lateral gauges were already encapsulated in their as-manufactured state and therefore did not require additional Mylar ${ }^{\circledR}$ protection. All components were glued together using slow curing Locite 0151 HYSOL ${ }^{\circledR}$ Epoxi-Patch ${ }^{\circledR}$ adhesive. Finally, before component assembly accurate sample 


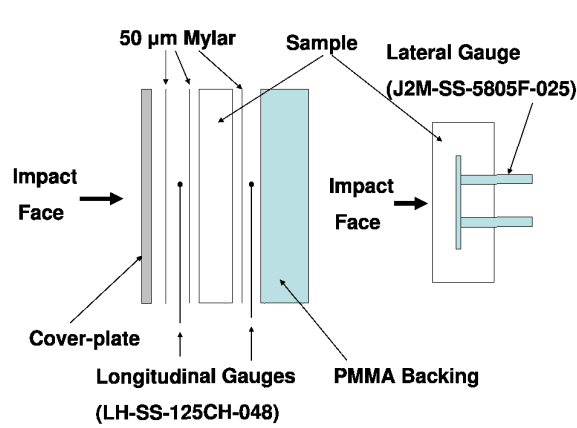

Fig. 2 Typical gauge/sample layouts: longitudinal on the left and lateral on the right

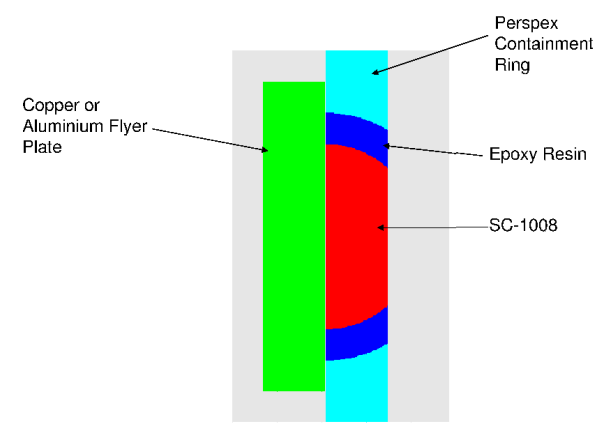

Fig. 3 Setup of the Autodyn ${ }^{\mathrm{TM}}$ model

thickness measurements were made using a micrometer. Typical gauge/sample arrangements are shown in Fig. 2.

\section{Computational Modeling}

A commercially available hydrocode (ANSYS Autodyn ${ }^{\mathrm{TM}}$ ) was employed to aid interpretation of recorded lateral stress profiles. Two-dimensionally axially symmetric models employing an Euler mesh with cell sizes of $100 \mu \mathrm{m}$ were constructed. Material models consisted of equations of state in conjunction with a Drucker-Prager strength model [21]. The initial Autodyn ${ }^{\mathrm{TM}}$ model setup is shown in Fig. 3.

\section{Material}

The phenolic resin investigated was Durite SC-1008. This was supplied by Lockheed Martin (UK) in $30 \mathrm{~mm}$ diameter spheres. The samples were sectioned with material taken from the centre of the sphere thereby giving an approximate thickness of $4 \mathrm{~mm}$ while keeping the diameter at approximately $30 \mathrm{~mm}$. Due to the small size of the SC-1008 phenolic resin target a containment ring was used to ensure the one-dimensionality of the

\begin{tabular}{|c|c|c|c|c|c|}
\hline $\begin{array}{c}\rho_{0} \\
\mathrm{gcm}^{-3}\end{array}$ & $\begin{array}{c}\mathrm{c}_{L} \\
\mathrm{~mm} \mu \mathrm{s}^{-1}\end{array}$ & $\begin{array}{c}\mathrm{c}_{S} \\
\mathrm{~mm} \mu \mathrm{s}^{-1}\end{array}$ & $\begin{array}{c}\mathrm{c}_{B} \\
\mathrm{~mm} \mu \mathrm{s}^{-1}\end{array}$ & $v$ & $\begin{array}{c}\mathrm{G} \\
\mathrm{GPa}\end{array}$ \\
\hline 1.18 & 2.67 & 1.38 & 2.14 & 0.36 & 2.24 \\
\hline
\end{tabular}

Table 1 Key elastic material properties of SC-1008

shock through the sample for the duration of the experiment. The containment ring arrangement with inserted resin (bonded in place with an epoxy resin) is shown schematically in Fig. 3.

The density of the SC-1008 phenolic resin was measured using a Micrometrics AccuPyc 1330 gas pycnometer. In addition longitudinal $\left(\mathrm{c}_{L}\right)$ and shear $\left(\mathrm{c}_{S}\right)$ sound velocities were measured using a $1 \mathrm{MHz}$ quartz transducer with a Panametrics 5077PR pulse receiver in the pulse-echo configuration. Key elastic material properties for SC-1008 are shown in Table 1, with the bulk sound speed $\mathrm{c}_{B}$ calculated using Eq. (6). The shear modulus $(\mathrm{G})$ of the material was calculated using Eq. (7).

$c_{B}=\sqrt{c_{L}^{2}-\frac{4}{3} c_{S}^{2}}$

$G=\rho_{0} c_{S}^{2}$

\section{Results and Discussion}

The experimental data for longitudinal and lateral shots is presented in Table 2. For the lateral shot data $\sigma_{x}$ was estimated from the known impact conditions. A typical longitudinal trace is shown in Fig. 4.

The rear gauge trace in Fig. 4 has been calibrated according to Eq. (8) [12], based on known impact conditions, to calculate the true stress in the SC-1008 rather than the backing PMMA (see Fig. 2). Both the front and re-calibrated rear surface longitudinal gauge traces exhibit very similar profiles. Following an initial rise (typically $\sim 50 \mathrm{~ns}$ duration, compared to $\sim 100 \mathrm{~ns}$ for the lateral traces shown later in Fig. 7) observed on shock arrival, an overshoot in stress combined with ringing results, with the frequency of ringing being 18 $\mathrm{MHz}$ suggesting an electrical effect. Following the initial overshoot in stress, a constant plateau known as the Hugoniot stress, is observed in both cases. The good agreement between the front and impedance matched rear Hugoniot stresses appears to be confirmation that the experiment was truly one-dimensional; validating the encapsulation technique employed. The Hugoniot stress is maintained until release waves from the rear of the flyer arrive ending the one-dimensional nature of 


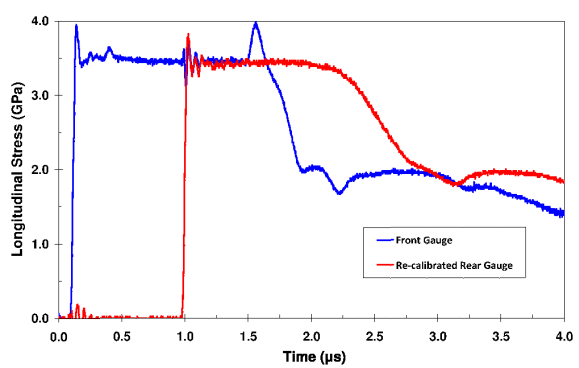

Fig. 4 Typical longitudinal gauge traces for SC-1008; impacted by a $5 \mathrm{~mm}$ Copper flyer at $960 \mathrm{~ms}^{-1}$

the shot. An apparent multi-stage release than occurs on both gauges, before eventual gauge failure.

$\sigma_{\text {Sample }}=\frac{1}{2} \frac{Z_{\text {Sample }}+Z_{\text {Backing }}}{Z_{\text {Backing }}} \sigma_{\text {Rear gauge }}$

Experimental results are shown graphically in the shock velocity-particle velocity plane in Fig. 5 and the pressure-volume plane in Fig. 6, along with literature data for other broadly similar polymeric materials. The RTM-6 data was obtained from Hazell et al. [11], with the alternative phenolic data from Carter and Marsh [8].

As seen in Fig. 5, the SC-1008 $\mathrm{U}_{S}-\mathrm{u}_{p}$ Hugoniot appears to have a non-linear nature within the particlevelocity regime investigated. Essentially, a polynomial curve of the type detailed in Eq. (2) produced the optimum fit to the experimental data. In this case, the polynomial coefficients for the best-fit were: $c_{0}=2.14$ $\mathrm{mm} \mu \mathrm{s}^{-1}, \mathrm{~S}_{1}=3.79$, and $\mathrm{S}_{2}=-1.68 \mu \mathrm{smm}^{-1}$. Such a non-linear response has been observed previously in other polymeric materials particularly at low particle velocities/pressures. For example, PMMA [7], polycarbonate replacement resin [22], and various epoxy resins [8], have all exhibited such behaviour. As discussed previously, such a non-linear response at low particle velocities has been attributed to the substantial difference in magnitude between backbone and inter-chain forces [8]. Essentially, weaker inter-chain forces are overcome during the initial stages of compression before stronger backbone covalent bonds can be affected. This then results in non-linear behaviour at low particle velocities, before a more conventional linear $\mathrm{U}_{S}-\mathrm{u}_{p}$ response is established as compression continues.

It is interesting to note that the lowest particlevelocity portion of the $\mathrm{U}_{S}-\mathrm{u}_{p}$ phenolic resin data found by Carter and Marsh [8], included in Fig. 5, is in very good agreement with the highest $\mathrm{u}_{p}$ data $\left(\mathrm{u}_{p}>0.6\right.$ $\mathrm{mm} \mu \mathrm{s}^{-1}$ ) measured for SC-1008 here. Given that Carter and Marsh found a linear relationship of the type shown in Eq. (1) for their phenolic resin over the pressure range between 4 and $20 \mathrm{GPa}$, and in line with the fact

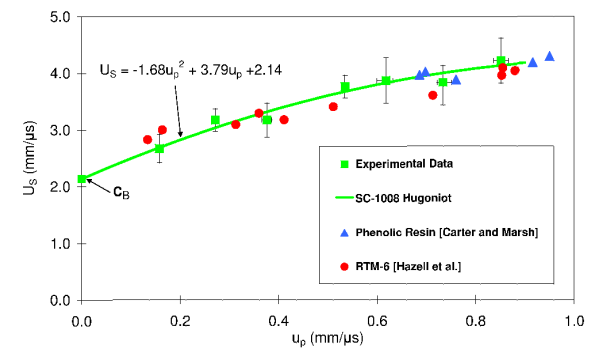

Fig. 5 Hugoniot of $\mathrm{SC}-1008$ in the $\mathrm{U}_{S}-\mathrm{u}_{p}$ plane with comparable resins $[8,11]$

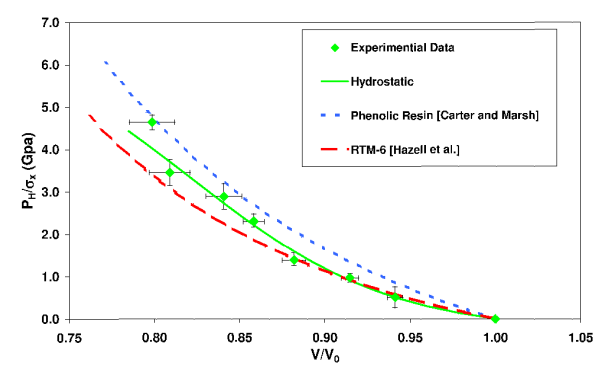

Fig. 6 Hugoniot of SC-1008 in the pressure-volume plane with comparable resins $[8,11]$

that $\mathrm{U}_{S}-\mathrm{u}_{p}$ relationships for epoxy resins are typically very similar independent of the precise resin tested [10, 11], this result suggests a two-stage $\mathrm{U}_{S}-\mathrm{u}_{p}$ behaviour in SC-1008 (and potentially phenolic resins in general). Initially, for $\mathrm{u}_{p}$ below $0.7-1.0 \mathrm{~mm} \mu \mathrm{s}^{-1}$ a non-linear polynomial Hugoniot will result, while at elevated impact pressures a linear response will be established. Interestingly, while slightly more linear in nature, there is also good general agreement between the included RTM 6 data from Ref. [11] and that for SC-1008.

In Fig. 6, $\mathrm{V} / \mathrm{V}_{0}$ (the normalised volume attained during the experiment on the abscissa) is plotted against $\mathrm{P}$. The use of $\mathrm{V} / \mathrm{V}_{0}$ allows direct comparisons with different materials. On the $\mathrm{P}-\mathrm{V} / \mathrm{V}_{0}$ plot the hydrostat is plotted according to Eq. (3). All of the experimental data with the exception of the highest value lies on the hydrostat. From Eq. (5) this implies that, initially at least, the SC-1008 possesses no strength under shock loading. However, above $4 \mathrm{GPa}$ deviation is seen from the hydrostat implying the material is starting to strengthen behind the shock front. This behaviour is similar to other polymeric materials including polycarbonate [23], polypropylene [24], polystyrene [24] and RTM-6 [11]. Further, the compressibility of the SC-1008 is initially the same as the epoxy resin RTM-6 until $\sim 1$ GPa. Above this point, the SC-1008 becomes less compressible compared to RTM-6. Additionally, the phenolic resin investigated here is more compressible than the phenolic resin investigated by Carter and Marsh. 


\begin{tabular}{|c|c|c|c|c|c|c|c|}
\hline Velocity & $\begin{array}{c}\text { Flyer Thickness/ } \\
\text { Material } \\
\mathrm{ms}^{-1}\end{array}$ & $\begin{array}{c}\mathrm{U}_{S} \\
\mathrm{~mm}\end{array}$ & $\begin{array}{c}\mathrm{u}_{p} \\
\mathrm{~mm} \mathrm{~s}^{-1}\end{array}$ & $\begin{array}{c}v \\
\mathrm{~mm} \mu \mathrm{s}^{-1}\end{array}$ & $\mathrm{~cm}^{-3} \mathrm{~g}^{-1}$ & $\begin{array}{c}\sigma_{x} / \mathrm{P} \\
\mathrm{GPa}\end{array}$ & $\begin{array}{c}\sigma_{y} \\
\mathrm{GPa}\end{array}$ \\
\hline 145 & $5 \mathrm{Cu}$ & $\cdots$ & $\cdots$ & 0.81 & 0.36 (Estimated) & 0.19 & 0.17 \\
\hline 200 & $10 \mathrm{Al}$ & 2.69 & 0.16 & 0.80 & 0.51 & $\cdots$ & $\cdots$ \\
\hline 320 & $10 \mathrm{Al}$ & $\cdots$ & $\cdots$ & 0.78 & 0.92 (Estimated) & 0.55 & 0.37 \\
\hline 350 & $10 \mathrm{Al}$ & 3.19 & 0.27 & 0.78 & 0.97 & $\cdots$ & $\cdots$ \\
\hline 485 & $10 \mathrm{Al}$ & 3.11 & 0.38 & 0.75 & 1.39 & $\cdots$ & $\cdots$ \\
\hline 500 & $10 \mathrm{Al}$ & $\cdots$ & $\cdots$ & 0.75 & 1.59 (Estimated) & 1.01 & 0.58 \\
\hline 600 & $10 \mathrm{Cu}$ & 3.77 & 0.53 & 0.73 & 2.31 & $\cdots$ & $\cdots$ \\
\hline 670 & $10 \mathrm{Cu}$ & $\cdots$ & $\cdots$ & 0.71 & 2.68 (Estimated) & 1.96 & 0.72 \\
\hline 810 & $10 \mathrm{Al}$ & 3.87 & 0.62 & 0.71 & 2.90 & $\cdots$ & $\cdots$ \\
\hline 865 & $10 \mathrm{Cu}$ & $\cdots$ & $\cdots$ & 0.69 & 3.65 (Estimated) & 2.61 & 1.04 \\
\hline 960 & $5 \mathrm{Al}$ & 3.88 & 0.73 & 0.69 & 3.46 & $\cdots$ & $\cdots$ \\
\hline 970 & $5 \mathrm{Cu}$ & 4.26 & 0.85 & 0.68 & 4.65 & $\cdots$ & $\cdots$ \\
\hline
\end{tabular}

Table 2 Experimental results obtained using longitudinal and lateral gauges

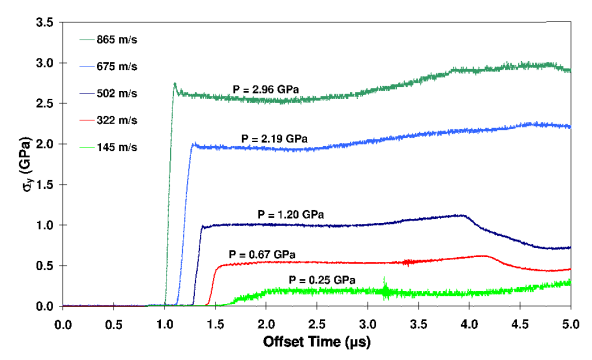

Fig. 7 Lateral gauge traces for SC-1008

Fig. 7 shows all of the lateral traces for the shots detailed in Table 2 plotted together with a recentered time base. The traces show an overshoot on the three higher pressure shots likely attributable to an electrical effect within the gauge. The pressure then stabalises to a plateau due to the lateral stress exerted on the material. A ramp-up is seen towards the end of the shot, and this has been computationally shown to be due to shock reflections from the interface between the edge of the SC1008 sample and the epoxy resin interlayer used in the containment process. Investigation of the source of this re-loading involved a series of Autodyn ${ }^{\mathrm{TM}}$ hydrocode models. This was achieved by using the setup explained in the computational modeling section and, for the case detailed here, the impact conditions of a $500 \mathrm{~ms}^{-1}$ shot employing a $10 \mathrm{~mm}$ aluminium flyer plate. In all cases a moving point gauge element was inserted $4 \mathrm{~mm}$ from the impact face of the sample. Additionally, this gauge was also radially offset from the impact axis by $4 \mathrm{~mm}$. The material equations of state used in the Autodyn ${ }^{\mathrm{TM}}$ model for aluminium, perspex and epoxy were obtained from the in-built Autodyn ${ }^{\mathrm{TM}}$ library. For the SC-1008 phenolic resin the experimentally determined polynomial equation of state employing the calculated values of density, $\mathrm{S}_{1}, \mathrm{~S}_{2}$ and $\mathrm{c}_{0}$ was used. The inelastic response was accounted for by the Drucker-Prager

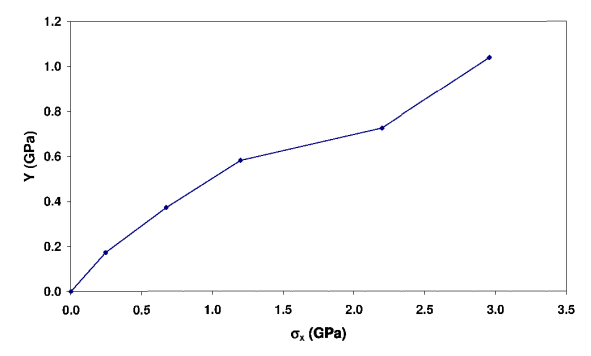

Fig. 8 Pressure-yield surface used in the Drucker-Prager strength model based on experimental measurements of $\sigma_{y}$

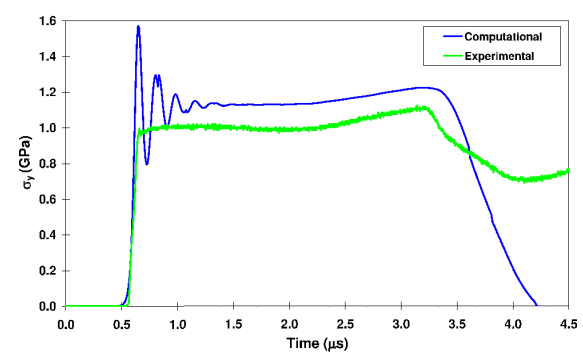

Fig. 9 Comparison of computational model and experimental lateral stresses (A $10 \mathrm{~mm}$ Aluminium flyer impacting at $508 \mathrm{~ms}^{-1}$

strength model, that employs a pressure-dependent yield surface [25-27]. Here Eq. (9) and Eq. (10) were used to calculate pressure and yield strength (Y) using the relevant $\sigma_{x}$ and $\sigma_{y}$ values from Table 2. The resultant yield surface e.g. the Drucker-Prager strength model is shown in Fig. 8.

$$
\begin{aligned}
& P_{\text {Average }}=\frac{\sigma_{x}+2 \sigma_{y}}{3} \\
& Y=2 \tau=\sigma_{x}-\sigma_{y}
\end{aligned}
$$

Fig. 9 shows reasonable agrement between the experimental data and computer simulation in terms of general behaviour, with a discrepancy in lateral stress 


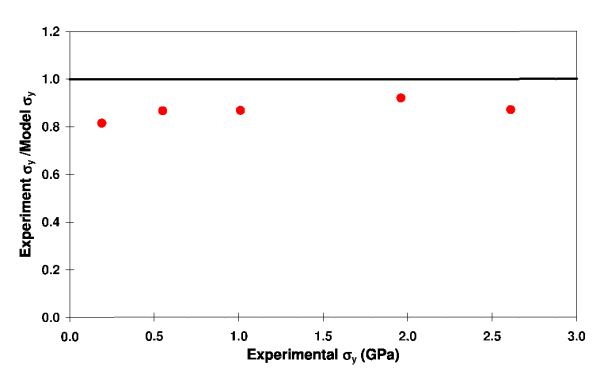

Fig. 11 Discrepancy between computational and experimental lateral stress

of about $0.1 \mathrm{GPa}$. Analysis of the temporal evolution of the sample showed that the increase in gradient occurring at about $1.5 \mu$ s after shock arrival was due to the shock reflection occurring at the SC-1008 and epoxy resin inter-layer. The timing and overall form of this reflection was seen to be linked to the geometry of the retained SC-1008 resin sample. It is worth noting that the arrival of this shock refection also marked the end of the one-dimensional behaviour in the sample. A sequence of frames showing the evolution of pressure in the model is presented in Fig. 10.

It should be noted that the gauge used in the model was a point element whereas in the experiments the lateral gauge was a $16 \mathrm{~mm}$ manganin wire element. Moving the gauge element in the computational model, the position at which 1D behaviour finished could be altered. By moving the gauge closer to the centre of the sample, the ramped behaviour was either delayed or became non-existent. Conversely, moving the gauge closer to the edge of the SC-1008 resin sample resulted in a more rapid loss of one-dimensionality in the gauge response. Due to the nature of the target confinement and geometry this response was not entirely unexpected.

The discrepancy noted above between the computational model and the experimental results in lateral gauges continues over the whole range of investigated shock pressures. In Fig. 11 the ratio of experimentally measured lateral stresses to computational results is plotted against measured lateral stress. If the Autodyn ${ }^{\mathrm{TM}}$ model matches up to the experimental results, a situation which should occur due to the experimental results being used in the Drucker-Prager strength model, this ratio should be close to unity. However, as shown in Fig. 11, this is clearly not the case. Instead the ratio is consistently around 0.8 to 0.9 - approximately 10 $20 \%$ lower than might be expected. Further, this discrepancy is observed to vary in a non-linear manner with measured lateral stress - an effect linked to the non-linear nature of the shear strength data employed in the Drucker-Prager strength model (as shown in Fig. $8)$.

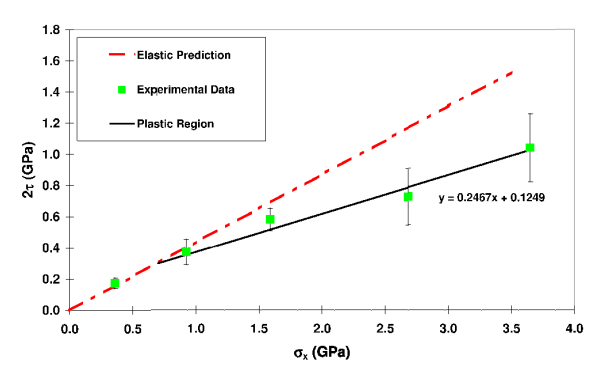

Fig. 12 Graph used for calculation of Hugoniot elastic limit

By comparing the yield strength of the material to an elastic prediction given by Eq. (11) the Hugoniot elastic limit can be ascertained. The Hugoniot elastic limit is the point at which the elastic-plastic transition occurs. Below the Hugoniot elastic limit the material behaves elastically and above this point it is plastic in nature. This approach is shown graphically in Fig. 12. From this the Hugoniot elastic limit was found to be $0.66 \pm 0.35 \mathrm{GPa}$. As indicated in Fig. 12 this relatively wide error simply arises due to the larger experimental errors encountered at elevated impact pressures. In addition, an approximate value for the Hugoniot elastic limit was found using Eq. (12). The value of $\mathrm{c}_{L}$ detailed in Table 1 was used in Eq. (2) to obtain the maximum possible particle velocity in the elastic regime (e.g. the $\mathrm{u}_{p}$ intercept of $\mathrm{c}_{L}$ with the $\mathrm{U}_{S}-\mathrm{u}_{p}$ Hugoniot shown in Fig. 5). This value, $\mathrm{u}_{H} \sim 0.1 \mathrm{~mm} \mu \mathrm{s}^{-1}$, gave a maximum value for the HEL of $0.3 \mathrm{GPa}$. This value, while low in comparison to the intersect shown in Fig. 12, lies just on the edge of the calculated error band. Given the aforementioned issues with calculating an HEL from the intercept of the predicted elastic and measured plastic shear strength curves in Fig. 12, the fact that these values are of similar orders of magnitude suggests that the average HEL of $0.66 \pm 0.35 \mathrm{GPa}$ from Fig. 12 is a reasonable approximation.

$2 \tau=\frac{1-2 v}{1-v} \sigma_{x}$

$\sigma_{H E L}=c_{L} \rho_{0} u_{H}$

Further, this reasonable agreement between the Hugoniot elastic limit values calculated (1) from the shear strength-impact stress plot in Fig. 12, and (2) directly from the ultrasonically measured elastic sound speed, appears to confirm the validity of the use of measured lateral stress values to calculate shear strengths. However, it should be noted that there is currently a degree of contention within the shock physics community with regards to the interpretation of such data. In particular, Winter and Harris [28] and Winter et al. [29] have recently highlighted the fact that there is often a difference between near and far field lateral stress response 


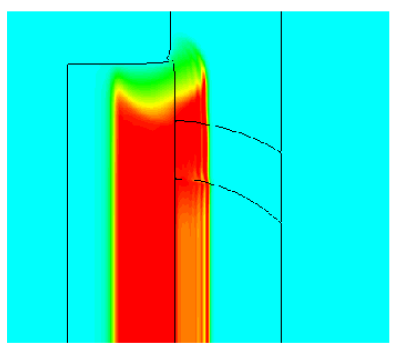

Time $=1.256 \mu \mathrm{s}$

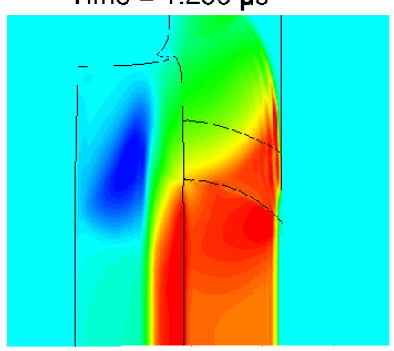

Time $=3.256 \mu \mathrm{s}$

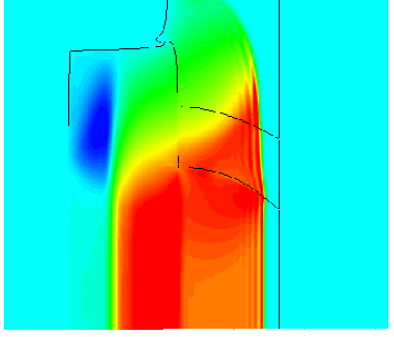

Time $=2.751 \mu \mathrm{s}$

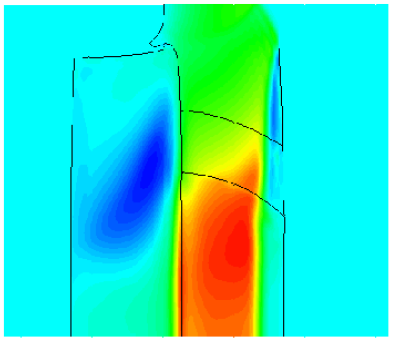

Time $=3.754 \mu \mathrm{s}$

Fig. 10 Behaviour of shock in Autodyn ${ }^{\mathrm{TM}}$ model

in materials due to the inherently intrusive nature of the encapsulated lateral manganin stress gauges employed. These authors employed both experiment and simulation to interrogate shock propagation through (a) a so-called matrix material, and (b) a matrix material with an embedded fluid layer - considered analogous to an encapsulated lateral gauge. The presence of the fluid layer was found to modify the nature of the shock front. In particular, behaviour on shock arrival and behind the shock front was strongly influenced by the relative fluid layer/matrix shock velocities. Slower shock velocity in the central fluid layer led to a ramp to a peak followed by a subsequent decrease in stress with time; whereas a faster fluid shock velocity was shown to result in a relatively slow initial ramp-up, with longitudinal stress subsequently increasing behind. In both cases comparison of experiment and simulation appeared to suggest that the gauge response tended towards the true material (rather than encapsulating matrix) stress with time. These findings suggest that care should be taken in the interpretation of lateral gauge traces. However, here - as shown in Fig. 7 - before the re-loading due to the target confinement highlighted in Fig. 9, there is only a relatively minor gradient in stress behind the shock. Consequently, lateral stresses measured by the epoxy-encapsulated lateral gauges are likely reasonably representative of far-field SC-1008 stresses. Further, in line with recent work by Appleby-Thomas et al. [30], it is likely that given the similarity in impedance and therefore shock velocity - between polymeric materials, minimal shock dispersion would have occurred in the epoxy lateral gauge interlayer. Nevertheless, the small resultant errors in measured lateral stress caused by these issues are likely to, at least partially, explain the discrepancy between the shear-strength and elastic sound-speed based predicted material elastic limits. Rosenberg and Partom [31] made the case that the HEL of polymers could be calculated using a pressure dependent yield surface which would alter Eq. (11). This would then result in different values for the elastic prediction used for calculating the HEL. However other authors have successfully used the model employed here hence we feel justified in using the approach presented in this paper.

In addition to the questions over the use of embedded lateral manganin stress gauges outlined above, the results presented here also draw attention to a further potential issue. Essentially, under the one-dimensional impact conditions of a plate impact experiment, because strain will be confined along the impact axis (defined as the x-axis), $\sigma_{y}$ and $\sigma_{z}$ will be equal. Consequently, by definition, the hydrostatic pressure $(\mathrm{P})$ will be given by Eq. (9). For a hydrodynamic material shear strength, $\tau$, will tend to zero. In such a situation Eq. (5) reduces to $\sigma_{x}=\mathrm{P}_{H}$. Substituting this result into Eq. (9) (taking $\mathrm{P}_{H}=\mathrm{P}_{\text {Average }}$ ), it can be shown that in a strength-less material $\sigma_{x}$ should be equal to $\sigma_{y}$. However, as can be seen in Table 2 , this is clearly not the case here; in fact, when calculated the ratio $\sigma_{y} / \sigma_{x}$ is observed to trend to about 0.8 at higher impact stresses, rather than trending to unity as would be expected. At lower stresses this is likely due to inaccuracies inherent 
in the employment of manganin stress gauges in such stress regimes [6]. However, at higher stresses this behaviour suggests a degree of material strength. This is despite the fact that SC-1008 has been shown to behave hydrodynamically (e.g. Fig. 6) at impact stresses $\leq 4.0$ GPa. Similar behaviour - with the ratio of $\sigma_{y} / \sigma_{x}$ tending to $<1$, despite observed hydrodynamic behaviour over the impact regime of interest, is observed elsewhere in the literature - e.g. Refs. [12,23,32-35]. Consequently, such a response may be indicative of a systematic calibration issue with lateral gauges in this class of materials. However, in order for such a conclusion to be reached a careful study of lateral gauge response in a variety of materials would be required - something outside the scope of this paper. However it is notable that there has been a wide adoption within the literature of the lateral gauge-based approach to estimation of material shear strength (albeit by a relatively limited number of authors), e.g. Refs $[12,23,32-38]$. In addition, as mentioned previously, in this study this approach has led to reasonable agreement with the HEL value calculated from the measured elastic sound speed. Consequently, the decision has been taken here to utilise the resultant calculated HEL. Nevertheless, the potential for inaccuracy due to gauge calibration issues is acknowledged.

\section{Conclusion}

The shock loading response of a phenolic resin SC1008 has been investigated using the flyer plate impact technique. A non-linear Hugoniot in the $\mathrm{U}_{S}-\mathrm{u}_{p}$ plane was found, following the equation $\mathrm{U}_{S}=2.49+$ $3.79 \mathrm{u}_{p}-1.68 \mathrm{u}_{p}^{2}$. In the $\mathrm{P}-\mathrm{V} / \mathrm{V}_{0}$ plane the experimental results closely followed the hydrostatic pressure suggesting minimal strengthening under shock loading. It was also found that the phenolic resin SC-1008 behaved in a similar manner to other polymeric materials that are used in comparable applications. An Autodyn ${ }^{\mathrm{TM}}$ model was used to help visualise the behaviour seen in the lateral stress traces. This behaviour was found to be due to the geometric nature of the resin sample. The pressure calculated from the model was shown to be close to the experimental data, albeit with a consistent discrepancy of $\sim 10 \%$. Finally, a Hugoniot elastic limit was calculated from both measured shear strength and ultrasonic sound speed data, with good agreement found leading to a value of $0.66 \pm 0.35 \mathrm{GPa}$ being assumed.

Acknowledgements The authors would like to acknowledge the support of the Institute of Shock Physics and AWE as well as Lockheed-Martin Insys for supplying the samples used for experimentation. We also wish to thank Mr Andrew Roberts for technical help with the experiments. British Crown Copyright $\mathrm{MOD} / 2011$.

\section{References}

1. Cowie JMG, Polymers: chemistry and physics of modern materials, 17-21. Blackie, Glasgow (1991).

2. Burrell RH, Barnes NK, Keightley PT, Millett JCF, Bourne NK, The response of a Carbon-fibre-phenolic resin Composite to One-dimensional shock loading, Americian Physical Society, 16th APS Conference, Poster presentation only (2009)

3. Fujiwara S, Explosive Technology for Generation of High Dynamic Pressure, In Shock Compression Technology and Material Science, Editor Sawaoka AB, KTK Scientific Publisher, Tokyo, 7-21 (1992)

4. Boustie M, Berthe L, de Resseguier T, Arrigoni M, Laser Shock Waves: Fundamentals and Applications, 1st International Symposium on Laser Ultrasonics: Science, Technology and Applications (2008)

5. Bourne NK, A $50 \mathrm{~mm}$ bore gas gun for dynamic loading of materials and structures, Meas. Sci. and Technol., 14, $273(2003)$

6. Meyers MA, Dynamic Behavior of Materials, 116-127 and 280. Wiley-Interscience Publication, New York (1994)

7. Barker LM, Hollenbach RE, Shock-Wave Studies of PMMA, Fused Silica, and Sapphire, J. Appl. Phys., 41, 4208-4226 (1970)

8. Carter WJ, Marsh SP, Hugoniot Equation of State of Polymers, Report LA-13006-MS, Los Alamos National Laboratory (1995)

9. Porter D, Gould PJ, Predictive nonlinear constitutive relations in polymers through loss history, Int. J. Solids Struct., 46, 1981-1993 (2009)

10. Munson DE, May RP, Dynamically Determined HighPressure Compressibilities of Three Epoxy Resin Systems, J. Appl. Phys., 43, 962-971 (1972)

11. Hazell PJ, Stennett C, Cooper G, The Shock and Release Behavior of an Aerospace-Grade Cured Aromatic Amine Epoxy Resin, Polym. Comp., 29, 1106-1110 (2008)

12. Appleby-Thomas G, Hazell P, Stennett C, The variation in lateral and longitudinal stress gauge response within an RTM 6 epoxy resin under one-dimensional shock loading, J. Mater. Sci., 44, 6187-6198 (2009)

13. Millett JCF, Bourne NK, Deas D, The equation of state of two alumina-filled epoxy resins, J. Phys. D: Appl. Phys., 38, 930 (2005)

14. Marsh SP, LASL Shock Hugoniot Data. University of California Press, California (1980)

15. Stennett C, Cooper GA, Hazell PJ, Appleby-Thomas GJ, Initiation of secondary explosives measured using embedded electromagnetic gauges, Shock Compression of Condensed Matter - 2009, 267-270. American Institute of Physics, Melville, NY (2009)

16. Volger TJ, Chhabildasa LC, Strength behavior of materials at high pressures, Int. J. Imp. Eng., 33, 812-825 (2006)

17. Rosenberg Z, Yaziv D, Partom Y, Calibration of foil-like manganin gauges in planar shock wave experiments, J. Appl. Phys., 51, 3702-3705 (1980)

18. Rosenberg Z, Brar NS, The influence of the elasto-plastic properties of piezoresistance gauges on their loadingunloading characteristics as lateral shock stress transducers, J. Appl. Phys., 77, 1443-1448 (1995) 
19. Rosenberg Z, Partom Y, Lateral stress measurement in shock-loaded targets with transverse piezoresistance gauges, J. Appl. Phys., 58, 3072-3076 (1985)

20. Millett JCF, Bourne NK, Rosenberg Z, On the analysis of transverse stress gauge data from shock loading experiments, J. Phys. D: Appl. Phys., 29, 2466 (1996)

21. Van Den Berg P, De Borst R, Hutink H, An Eulerean finite element model for penetration in layered soil, International J. Numer. Anal. Methods in Geomec., 20, 865886 (1996)

22. Appleby-Thomas GJ, Hazell PJ, Stennett C, Cooper G, Cleave R, The dynamic behaviour of a modified polyurethane resin, DYMAT 2009- 9th International Conference on the Mechanical and Physical Behaviour of Materials under Dynamic Loading, 2, 1081-1087 (2009)

23. Millett J, Bourne N, Shock and release of polycarbonate under one-dimensional strain, J. Mater. Sci., 41, 16831690 (2006)

24. Millett JCF, Bourne NK, The shock induced equation of state of three simple polymers, J. Phys. D: Appl. Phys., 37, 2901 (2004)

25. Park SW, Xia Q, Zhou M, Dynamic behavior of concrete at high strain rates and pressures: II. numerical simulation, Int. J. Imp. Eng., 25, 887-910 (2001)

26. Tham CY, Reinforced concrete perforation and penetration simulation using AUTODYN-3D, Finite Elements in Analysis and Design, 41, 1401-1410 (2005)

27. Ma GW, Hao H, Zhou YX, Modeling of wave propagation induced by underground explosion, Comp. Geotech., 22, 283-303 (1998)

28. Winter RE, Harris EJ, Simulations of embedded lateral stress gauge profiles in shocked targets, J. Phys. D: Appl. Phys., 41, 035503 (2003)

29. Winter RE, Owen GD, Harris EJ, Experimental measurement of stress perturbations caused by lateral gauges, J. Phys. D: Appl. Phys., 41, 202006 (2008)

30. Appleby-Thomas GJ, Hazell PJ, Wilgeroth JM, Wood $\mathrm{DC}$, On the interpretation of lateral manganin gauge stress measurements in polymers, J. Appl. Phys., 108, 033524 (2010)

31. Rosenberg Z, Partom Y, Accounting for the Hugoniot elastic limits of polymers by using pressure-dependent yield criterion, J. Appl. Phys., 76, 1935-1936 (1994)

32. Millett JCF, Bourne NK, Barnes NK, The behavior of an epoxy resin under one-dimensional shock loading, J. Appl. Phys., 92, 6590-6594 (2002)

33. Bourne NK, Millett JCF, Goveas SG, The shock response of polyoxymethylene and polyethylene, J. Phys. D: Appl. Phys., 40, 5714 (2007)

34. Millett JCF, Bourne NK, GrayIII GT, Cooper G, On the shock response of polychloroprene, Shock Compression of Condensed Matter - 2001, 131-134, American Institute of Physics, Melville, NY (2002)

35. Millett JCF, Bourne NK, Gray III GT, The response of polyether ether ketone to one-dimensional shock loading, J. Phys. D: Appl. Phys., 37, 942 (2004)

36. Pickup IM, Millett JCF, Bourne NK, The shock behaviour of a $\mathrm{SiO} 2-\mathrm{Li} 2 \mathrm{O}$ transparent glass-ceramic armour material, Shock Compression of Condensed Matter - 2003, American Institute of Physics, Melville NY, 751-754 (2004)

37. Ragford DD, The inelastic behavior and failure of dense glass under shock loading to $15 \mathrm{GPa}$, J. Appl. Phys., 98, $063504(2005)$

38. Appleby-Thomas GJ, Hazell PJ, A study on the strength of an armour-grade aluminum under high strain-rate loading, J. Appl. Phys., 107, 123508 (2010)
39. Bourne NK, Millett JCF, The high-rate response of an elastomer, Proc. R. Soc. London. Series A: Math., Phys. Eng. Sci., 459, 597-576 (2003) 